\title{
Activating Hidden Metrological Usefulness
}

\author{
Géza Tóth $\odot^{*}$ \\ Department of Theoretical Physics, University of the Basque Country UPV/EHU, P.O. Box 644, E-48080 Bilbao, Spain, \\ Donostia International Physics Center (DIPC), P.O. Box 1072, E-20080 San Sebastián, Spain, \\ IKERBASQUE, Basque Foundation for Science, E-48013 Bilbao, Spain, \\ and Wigner Research Centre for Physics, Hungarian Academy of Sciences, P.O. Box 49, H-1525 Budapest, Hungary \\ Tamás Vértesi® ${ }^{\dagger}$ \\ MTA Atomki Lendület Quantum Correlations Research Group, Institute for Nuclear Research, \\ Hungarian Academy of Sciences, P.O. Box 51, H-4001 Debrecen, Hungary \\ Paweł Horodecki \\ International Centre for Theory of Quantum Technologies, University of Gdańsk, Wita Stwosza 63, 80-308 Gdańsk, Poland \\ and Faculty of Applied Physics and Mathematics, National Quantum Information Centre, Gdansk University of Technology, \\ Gabriela Narutowicza 11/12, 80-233 Gdańsk, Poland \\ Ryszard Horodecki \\ International Centre for Theory of Quantum Technologies, University of Gdańsk, Wita Stwosza 63, 80-308 Gdańsk, Poland \\ and Institute of Theoretical Physics and Astrophysics, National Quantum Information Centre, Faculty of Mathematics, \\ Physics and Informatics, University of Gdańsk, Wita Stwosza 57,80-308 Gdańsk, Poland
}

(Received 27 November 2019; accepted 8 June 2020; published 7 July 2020)

\begin{abstract}
We consider bipartite entangled states that cannot outperform separable states in any linear interferometer. Then, we show that these states can still be more useful metrologically than separable states if several copies of the state are provided or an ancilla is added to the quantum system. We present a general method to find the local Hamiltonian for which a given quantum state performs the best compared to separable states. We obtain analytically the optimal Hamiltonian for some quantum states with a high symmetry. We show that all bipartite entangled pure states outperform separable states in metrology. Some potential applications of the results are also suggested.
\end{abstract}

DOI: 10.1103/PhysRevLett.125.020402

Entanglement lies at the heart of quantum mechanics and plays an important role in quantum information theory [1]. Recently, it has been realized that entanglement can be a useful resource in very general metrological tasks. By using entangled states it is possible to overcome the shot-noise limit, corresponding to classical interferometers, in the precision of parameter estimation [2-7]. On the other hand, separable states, i.e., states without entanglement cannot overcome the classical limit. It has even been shown that quantum states with a very weak form of entanglement, called bound entanglement [8-10], can also be metrologically useful in this sense $[11,12]$. However, there are highly entangled states that are not useful for metrology [13].

In what sense is metrological usefulness the property of the quantum state? It is clear that, starting from many

Published by the American Physical Society under the terms of the Creative Commons Attribution 4.0 International license. Further distribution of this work must maintain attribution to the author(s) and the published article's title, journal citation, and DOI. entangled quantum states that are not useful for metrology, with local operations and classical communication (LOCC) it is possible to distill singlets, which are metrologically useful. This finding is almost trivial, as metrological "uselessness" is not conserved by LOCC operations. On the other hand, in quantum metrology experiments most LOCC operations are typically not possible. Here, we investigate how metrological usefulness can change in the two simplest cases very relevant in practice: we consider adding an ancilla to a single copy of the bipartite quantum state. We also consider providing two copies of the state [14]. These two scenarios follow the spirit in which the activation of bound entanglement and nonlocality has been studied [10,15-17] (see Fig. 1).

In this Letter, we show that some bipartite entangled quantum states that are not useful in linear interferometers become useful in the cases mentioned above. These findings are quite surprising: including uncorrelated ancilla qubits can make a state metrologically useful. To support our claims, we present a general method to find the local Hamiltonian for which a given bipartite quantum state 


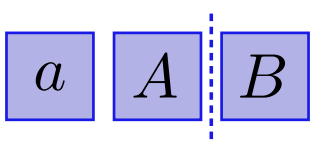

(a)

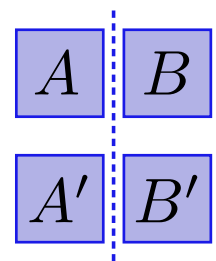

(b)
FIG. 1. (a) An ancilla (" $a$ ") is added to bipartite state $\varrho_{A B}$. (b) An additional copy or a different state is added to the state. In both cases, a new bipartite state is obtained, where the two parties are separated by a dashed line.

provides the largest gain compared to separable states. Note that this task is different, and in a sense more complex, than maximizing the quantum Fisher information. The reason is that by changing the Hamiltonian, the sensitivity achievable by separable states can also change.

Quantum Fisher information.-Before discussing our main results, we review some of the fundamental relations of quantum metrology. A basic metrological task in a linear interferometer is estimating the small angle $\theta$ for a unitary dynamics $U_{\theta}=\exp (-i \mathcal{H} \theta)$, where the Hamiltonian is the sum of local terms. That is, all local terms act within the subsystem and there are no interactions between the subsystems. In particular, for bipartite systems it is

$$
\mathcal{H}=\mathcal{H}_{1}+\mathcal{H}_{2},
$$

where $\mathcal{H}_{n}$ are single-subsystem operators. The precision is limited by the Cramér-Rao bound as [18-21]

$$
(\Delta \theta)^{2} \geq \frac{1}{m \mathcal{F}_{Q}[\varrho, \mathcal{H}]},
$$

where $m$ is the number of independent repetitions, and the quantum Fisher information, a central quantity in quantum metrology is defined by the formula [18]

$$
\mathcal{F}_{Q}[\varrho, \mathcal{H}]=2 \sum_{k, l} \frac{\left(\lambda_{k}-\lambda_{l}\right)^{2}}{\lambda_{k}+\lambda_{l}}|\langle k|\mathcal{H}| l\rangle|^{2} .
$$

Here, $\lambda_{k}$ and $|k\rangle$ are the eigenvalues and eigenvectors, respectively, of the density matrix $\varrho$, which is used as a probe state for estimating $\theta$.

Metrological usefulness of a quantum state.-We call a quantum state metrologically useful, if it can outperform separable states in some metrological task, i.e., if

$$
\mathcal{F}_{Q}[\varrho, \mathcal{H}]>\max _{\varrho_{\text {sep }}} \mathcal{F}_{Q}\left[\varrho_{\text {sep }}, \mathcal{H}\right]=: \mathcal{F}_{Q}^{(\text {sep })}(\mathcal{H})
$$

It is an intriguing task to find the operator $\mathcal{H}$, for which a given state performs the best compared to separable states.
For that we define the metrological gain compared to separable states by

$$
g_{\mathcal{H}}(\varrho)=\mathcal{F}_{Q}[\varrho, \mathcal{H}] / \mathcal{F}_{Q}^{(\text {sep })}(\mathcal{H}) .
$$

We are interested in the quantity

$$
g(\varrho)=\max _{\text {local } \mathcal{H}} g_{\mathcal{H}}(\varrho),
$$

where a local Hamiltonian is just the sum of single system Hamiltonians as in Eq. (1). The maximization task looks challenging since we have to maximize a fraction, where both the numerator and the denominator depend on the Hamiltonian. (See the Supplemental Material for basic properties of the metrological gain [22].)

Maximally entangled state.-As we have mentioned, it is a difficult task to obtain $g(\varrho)$ and the optimal local Hamiltonian for any $\varrho$. As a first step, we consider the $d \times d$ maximally entangled state, which is defined as

$$
\left|\Psi^{(\mathrm{me})}\right\rangle=\frac{1}{\sqrt{d}} \sum_{k=1}^{d}|k\rangle|k\rangle .
$$

Due to the symmetry of the state, the optimal Hamiltonian can straightforwardly be obtained as

$$
\mathcal{H}^{(\mathrm{me})}=D \otimes \mathbb{1}+\mathbb{1} \otimes D,
$$

where the diagonal matrix $D$ is given as

$$
D=\operatorname{diag}(+1,-1,+1,-1, \ldots) .
$$

The details are given in the Supplemental Material [22]. For the $3 \times 3$ case, we consider the noisy quantum state

$$
\varrho_{A B}^{(p)}=(1-p)\left|\Psi^{(\mathrm{me})}\right\rangle\left\langle\Psi^{(\mathrm{me})}\right|+p \mathbb{1} / d^{2},
$$

which is useful if [22]

$$
p<\frac{25-\sqrt{177}}{32} \approx 0.3655 .
$$

(See the Supplemental Material for the definition of the related notion of robustness of metrological usefulness [22].)

Activation by an ancilla qubit.-Now we consider the previous state, after a pure ancilla qubit is added

$$
\varrho^{(\mathrm{anc})}=|0\rangle\left\langle\left. 0\right|_{a} \otimes \varrho_{A B}^{(p)} .\right.
$$

The setup is depicted in Fig. 1(a). Then, with the operator

$$
\mathcal{H}^{(\mathrm{anc})}=1.2 C_{a A} \otimes \mathbb{1}_{B}+\mathbb{1}_{a A} \otimes D_{B},
$$

where an operator acting on the ancilla and $A$ is 


$$
C_{a A}=\frac{9}{20}\left(2 \sigma_{x}+\sigma_{z}\right)_{a} \otimes|0\rangle\left\langle\left. 0\right|_{A}+\mathbb{1}_{a} \otimes\left(|2\rangle\left\langle\left. 2\right|_{A}-\mid 1\right\rangle\left\langle\left. 1\right|_{A}\right),\right.\right.
$$

we have $g_{\mathcal{H}^{\text {(anc) }}}\left(\varrho^{\text {(anc) })}\right)>1$ if $p<0.3752$ [cf. Eq. (11)]. Hence larger part of the noisy maximally entangled states are useful in the case with the ancilla.

Activation by adding extra copies.-We consider now two copies of the noisy $3 \times 3$ maximally entangled state

$$
\varrho^{(\mathrm{tc})}=\varrho_{A B}^{(p)} \otimes \varrho_{A^{\prime} B^{\prime}}^{(p)}
$$

The setup is shown in Fig. 1(b). Then, with the two-copy operator

$$
\mathcal{H}^{(\mathrm{tc})}=D_{A} \otimes D_{A^{\prime}} \otimes \mathbb{1}_{B B^{\prime}}+\mathbb{1}_{A A^{\prime}} \otimes D_{B} \otimes D_{B^{\prime}},
$$

we have $g_{\mathcal{H}^{(\mathrm{tc})}}\left(\varrho^{(\mathrm{tc})}\right)>1$ if $p<0.4164$ [cf. Eq. (11)]. Hence larger part of the noisy maximally entangled states are useful in the two-copy case, than with a single copy. So far we have studied the $3 \times 3$ case. For the $2 \times 2$ case, see the Supplemental Material [22].

Observation 1: In summary, we have just shown that there are bipartite states with the following properties. (i) They are not more useful than separable states considering any local Hamiltonian. (ii) By adding an ancilla or two copies, they are more useful than separable states for some local Hamiltonian. For the case of an added ancilla, the new subsystems are now $a A$ and $B$, and the Hamiltonian contains interactions between the ancilla $a$ and $A$. In the two-copy case, the new subsystems are $A A^{\prime}$ and $B B^{\prime}$, and the Hamiltonian contains interactions between $A$ and $A^{\prime}$, and between $B$ and $B^{\prime}$. Note that in both cases, the extra interactions increase the metrological capabilities of separable states. Still, simple algebra shows that in both cases the metrological gain can stay the same or can increase, but cannot decrease [22].

So far, we exploited the symmetries of quantum states to obtain the Hamiltonian leading to the largest metrological gain. We now present a general method to compute $g(\varrho)$ numerically.

Method for finding the optimal Hamiltonian.-We need to maximize $\mathcal{F}_{Q}[\varrho, \mathcal{H}]$ over $\mathcal{H}$ for a given $\varrho$. However, since it is convex in $\mathcal{H}$, maximizing it over $\mathcal{H}$ is a difficult task. Instead of the quantum Fisher information, let us consider the error propagation formula

$$
(\Delta \theta)^{2}{ }_{M}=\frac{(\Delta M)^{2}}{\langle i[M, \mathcal{H}]\rangle^{2}},
$$

which provides a bound on the quantum Fisher information [22,36-38]

$$
\mathcal{F}_{Q}[\varrho, \mathcal{H}] \geq 1 /(\Delta \theta)_{M}^{2}
$$

We will now minimize Eq. (17).

Observation 2: The error propagation formula given in Eq. (17) can be minimized over $\mathcal{H}$ for a given $M$ and $\varrho$ as follows.

Proof.-Simple algebra yields

$$
\langle i[M, \mathcal{H}]\rangle=\operatorname{Tr}\left(A_{1} \mathcal{H}_{1}\right)+\operatorname{Tr}\left(A_{2} \mathcal{H}_{2}\right),
$$

where $A_{n}=\operatorname{Tr}_{\{1,2\} \backslash n}(i[\varrho, M])$ are operators acting on a single subsytem. Hence, we have to maximize Eq. (19) over $\mathcal{H}_{1}$ and $\mathcal{H}_{2}$. We choose the constraints

$$
c_{n} \mathbb{1} \pm \mathcal{H}_{n} \geq 0,
$$

where $n=1,2$ and $c_{n}>0$ is some constant. This way we make sure that $\sigma_{\min }\left(\mathcal{H}_{n}\right) \geq-c_{n}$, and $\sigma_{\max }\left(\mathcal{H}_{n}\right) \leq+c_{n}$, for $n=1,2$, where $\sigma_{\min }(X)$ and $\sigma_{\max }(X)$ denote the smallest and largest eigenvalues of $X$. The optimal $\mathcal{H}_{n}$ is the one that maximizes $\operatorname{Tr}\left(A_{n} \mathcal{H}_{n}\right)$ under these constraints. It can straightforwardly be obtained as

$$
\mathcal{H}_{n}^{(\mathrm{opt})}=U_{n} \tilde{D}_{n} U_{n}^{\dagger},
$$

where the eigendecompisition of $A$ is given as $A_{n}=U_{n} D_{n} U_{n}^{\dagger}$ and $\left(\tilde{D}_{n}\right)_{k, k}=c_{n} s\left[\left(D_{n}\right)_{k, k}\right]$, where $s(x)=1$ if $x \geq 0$, and -1 otherwise. Clearly, $\mathcal{H}_{n}^{(\mathrm{opt})}$ has the same eigenvectors as $A_{n}$ and has only eigenvalues $+c_{n}$ and $-c_{n}$.

We already know how to optimize $\mathcal{H}$ for a given $M$. However, how do we find the optimal $M$ ? This can be done with the well-known formula for the symmetric logarithmic derivative [21]

$$
M_{\mathrm{opt}}=2 i \sum_{k, l} \frac{\lambda_{k}-\lambda_{l}}{\lambda_{k}+\lambda_{l}}|k\rangle\langle l|\langle k|\mathcal{H}| l\rangle
$$

For a given $\mathcal{H}$, the error propagation formula given in Eq. (17) is minimized for $M=M_{\text {opt }}$ [22,37].

Iterative method.-We can now construct the following procedure for minimizing Eq. (17). First we choose a random $M$. Then, repeat the following two steps. (Step 1) Determine the optimal $\mathcal{H}$ for a given $M$ using Observation 2. (Step 2) Determine the optimal $M$ for a given $\mathcal{H}$ using Eq. (22). A see-saw procedure similar in spirit has been used to make the optimization of the metrological performance over density matrices in Refs. [12,39,40].

After several iterations of the two steps above, we obtain the maximal quantum Fisher information over a certain set of Hamiltonians. Based on that, we can calculate the quantity 


$$
g_{c_{1}, c_{2}}(\varrho)=\max _{\mathcal{H}_{1}, \mathcal{H}_{2}} \frac{\mathcal{F}_{Q}\left(\varrho, \mathcal{H}_{1} \otimes \mathbb{1}+\mathbb{1} \otimes \mathcal{H}_{2}\right)}{\mathcal{F}_{Q}^{(\text {sep })}\left(c_{1}, c_{2}\right)},
$$

where we assumed that $\mathcal{H}_{n}$ are constrained with Eq. (20). The separable limit for Hamiltonians of the form (1) is $[12,41]$

$$
\mathcal{F}_{Q}^{(\text {sep })}(\mathcal{H})=\sum_{n=1,2}\left[\sigma_{\max }\left(\mathcal{H}_{n}\right)-\sigma_{\min }\left(\mathcal{H}_{n}\right)\right]^{2}
$$

which leads to $\mathcal{F}_{Q}{ }^{(\mathrm{sep})}\left(c_{1}, c_{2}\right)=4\left(c_{1}^{2}+c_{2}^{2}\right)$. Then, the gain can be expressed as

$$
g(\varrho)=\max _{c_{2}} g_{c_{1}, c_{2}}(\varrho)
$$

where the optimization is only over $c_{2}$, and, without the loss of generality, we set $c_{1}=1$. The optimal $c_{2}$ can be obtained from an analytical formula [22]. Hence we computed the maximum of the fraction (5) for local Hamiltonians.

We now stress the following. If we determine the optimal $\mathcal{H}$ for a given $M$ using Observation 2, the eigenvalues of the optimal $\mathcal{H}_{n}$ satisfying Eq. (20) are $\pm c_{n}$. Let us assume the contrary. Let us assume that for a state $Q$ and for given $c_{1}, c_{2}$ we know the optimal $\mathcal{H}_{1}$ and $\mathcal{H}_{2}$, and $\mathcal{H}_{n}$ fulfill Eq. (20), but not all eigenvalues are $\pm c_{n}$. We observe that $\langle i[M, \mathcal{H}]\rangle$ is a linear function of the eigenvalues of $\mathcal{H}_{n}$, thus it takes its maximum at the eigenvalues corresponding to the boundary of the allowed region. Hence, we can always replace the eigenvalues of $\mathcal{H}_{n}$ by $\pm c_{n}$ such that $\langle i[M, \mathcal{H}]\rangle$ will not decrease, and $1 /(\Delta \theta)^{2}{ }_{M}$ will not decrease either.

Using the numerical method above, we obtain a slightly larger value for the noise bounds of metrological usefulness for the state with an ancilla, (12). $g\left(\varrho^{(\text {anc })}\right)>1$ if $p<0.3941$. The same is true for the case of the two copies of the noisy maximally entangled state, (15). We obtain $g\left(\varrho^{(\mathrm{tc})}\right)>1$ if $p<0.4170$.

For states with a high symmetry, such as isotropic states $[42,43]$, and Werner states [44], we obtained the optimal Hamiltonian analytically and determined the subset of these states that are metrologically useful [22]. We also used that to verify our numerical methods.

Activation of a bound entangled state by a separable state.-While bound entangled or nondistillable states [8,9] are considered weakly entangled, they can share many properties with highly entangled states. For example, there are bound entangled states that can reach the Heisenberg scaling in metrological applications [11]. It has also been shown that bipartite bound entangled states, which have a positive semidefinite partial transposition (PPT), can be useful for metrology [12]. Moreover, bipartite PPT entangled states can even have a high Schmidt rank [45].

Let us now consider a PPT entangled state $\varrho_{A B}^{(\mathrm{PPT})}$ that is not useful for quantum metrology. Then, we look for a separable state $\varrho^{(\mathrm{sep})}$ such that $\varrho_{A B}^{(\mathrm{PPT})} \otimes \varrho_{A^{\prime} B^{\prime}}^{(\mathrm{sep})}$ becomes useful. Hence, in this case we have to optimize not only over $\mathcal{H}, M$, but also over the separable state. Simple convexity arguments show that the maximum is taken when we have a pure product state, $\varrho_{A^{\prime} B^{\prime}}^{(\text {sep }}=\varrho_{A^{\prime}}^{(\text {anc })} \otimes \varrho_{B^{\prime}}^{(\text {anc })}$, which corresponds to two ancillas at the two parties. In fact, even a single ancilla qubit is sufficient for activation.

Activation of a PPT entangled state by an ancilla qubit.-We now consider a PPT entangled state, which is not useful metrologically, and $g\left(\varrho_{A B}\right)=1$. However, with an ancilla it becomes useful, $g\left(\varrho_{(a A)(B)}\right)>1$. We show here examples for $d \times d$ dimensional PPT states found in Ref. [12] for odd dimensions $d$ up to $d \leq 11$. See Table I for the numerical results.

Note that here we fixed $c_{i}=1$ for the coefficients of the local Hamiltonians $\mathcal{H}_{i}, i=1,2$. However, numerics suggests that optimization over $c_{i}$ does not help to increase $g$ in the case of two ancillas (last column), due to the permutational symmetry of the states. Optimization over $c_{i}$ helps only marginally in the case of one ancilla (third column). For instance, in the case of $d=7$, the $g$ value raises from 1.0096 (corresponding to $c_{2}=1$ ) to 1.0098 (corresponding to $c_{2} \simeq 1.034$ ) if we optimize over $c_{2}$.

Entanglement detection.-Our method can be used for entanglement detection. It identifies the Hamiltonians with which a given quantum state performs better than separable states and hence it is detected as entangled. If we add ancillas or extra copies of the quantum state, the criterion can be even more powerful.

Random states.-We can use our method to determine the distribution of metrological usefulness of random pure or mixed states of a given size. For instance, for $3 \times 3$ systems, pure states typically are close to be maximally useful, while this is not the case if we look for the usefulness with respect to a given Hamiltonian.

TABLE I. Activation of the metrological usefulness found numerically in two-qudit systems. (First column) Local dimension $d$, where $d$ is odd. For even $d$ up to $d \leq 11$, we did not find activation in the examples of PPT two-qudit states considered. (Second column) White noise fractions of $p^{*}$ added to the PPT states given by Ref. [12] such that $g_{1,1}\left(\varrho_{A B}\right)=1.0000$, that is, they are not useful metrologically. (Third column) Metrological gain after an ancilla is added to Alice's system, $g_{1,1}\left(\varrho_{(a A)(B)}\right)$. The states become useful as demonstrated by $g_{1,1}\left(\varrho_{(a A)(B)}\right)>1$. (Fourth column) Metrological gain after a further ancilla is added to Bob's system, $g_{1,1}\left(Q_{(a A)(B b)}\right)$. The state becomes even more useful metrologically.

\begin{tabular}{lccc}
\hline \hline$d$ & $p^{*}$ & Gain with one ancilla & Gain with two ancillas \\
\hline 3 & 0.0006 & 1.0007 & 1.0011 \\
5 & 0.0960 & 1.0094 & 1.0190 \\
7 & 0.1377 & 1.0096 & 1.0195 \\
9 & 0.1631 & 1.0090 & 1.0181 \\
11 & 0.1807 & 1.0081 & 1.0165 \\
\hline \hline
\end{tabular}


For the numerical result, please see the Supplemental Material [22].

Usefulness of entangled bipartite pure states. - Next we will consider the usefulness of bipartite pure states.

Observation 3: All entangled bipartite pure states are metrologically useful. (For the two-qubit case, see Ref. [13].)

Proof.-Let us consider a pure state with a Schmidt decomposition

$$
|\Psi\rangle=\sum_{k=1}^{s} \sigma_{k}|k\rangle_{A}|k\rangle_{B}
$$

where $s$ is the Schmidt number, and the real positive $\sigma_{k}$ Schmidt coefficients are in a descending order. We define

$$
\mathcal{H}_{A}=\sum_{n=1,3,5, \ldots, \tilde{s}-1}|+\rangle\left\langle+\left.\right|_{A, n, n+1}-\mid-\right\rangle\left\langle-\left.\right|_{A, n, n+1},\right.
$$

where $\tilde{s}$ is the largest even number for which $\tilde{s} \leq s$, and

$$
| \pm\rangle_{A, n, n+1}=\left(|n\rangle_{A} \pm|n+1\rangle_{A}\right) / \sqrt{2} .
$$

We define $\mathcal{H}_{B}$ in a similar manner. We also define the collective Hamiltonian

$$
\mathcal{H}_{A B}=\mathcal{H}_{A} \otimes \mathbb{1}+\mathbb{1} \otimes H_{B}
$$

Then, we have $\left\langle\mathcal{H}_{A B}\right\rangle_{\Psi}=0$. Direct calculation yields

$$
\mathcal{F}_{Q}\left[|\Psi\rangle, \mathcal{H}_{A B}\right]=4\left(\Delta \mathcal{H}_{A B}\right)^{2} \Psi=8 \sum_{n=1,3,5, \ldots, \tilde{s}-1}\left(\sigma_{n}+\sigma_{n+1}\right)^{2},
$$

which is larger than the separable bound, $\mathcal{F}_{Q}^{(\text {sep })}=8$, whenever the Schmidt rank is larger than 1. For even $s$, this can be seen noting that

$$
\mathcal{F}_{Q}\left[|\Psi\rangle, \mathcal{H}_{A B}\right]>8 \sum_{n=1}^{s} \sigma_{n}^{2}
$$

holds, where we used Eq. (30) to evaluate the left-hand side of Eq. (31), and we also took into account that $\sigma_{n}>0$ for $n=1,2,3, \ldots$, and $\sum_{n=1}^{s} \sigma_{n}^{2}=1$. For odd $s$, we need that

$$
\mathcal{F}_{Q}\left[|\Psi\rangle, \mathcal{H}_{A B}\right] \geq 8\left(\sum_{n=1}^{s-1} \sigma_{n}^{2}+2 \sigma_{1} \sigma_{2}\right)>8 \sum_{n=1}^{s} \sigma_{n}^{2}
$$

holds, where we used that $\sigma_{1} \sigma_{2}>\sigma_{s}^{2}$.

We can even consider several copies of a quantum state. In the Supplemental Material, we prove that for infinite number of copies of entangled pure quantum states the metrological gain is maximal [22].

Conclusions.-We showed that entangled quantum states that cannot outperform separable states in any linear interferometer can still be more useful than separable states, if several copies of them are considered or an ancilla is added to the system. This is a surprising result which shows that the relationship between quantum metrology and the structure of quantum states requires further study. We presented a method to find the Hamiltonian for carrying out metrology in a linear interferometer with a given quantum state that provides the largest gain compared to the precision achievable by separable states. In the Letter we considered bipartite problems, thus it would be important to extend this approach to multipartite systems and examine the scaling of the metrological gain for noisy quantum states. It would be also interesting to look for application in entanglement detection [1], and witnessing the dimension of quantum systems [46-49], where the results of the preliminary analysis seem to be promising. (See the Supplemental Material [22].)

We thank I. Apellaniz, D. Gross, O. Gühne, S. Imai, M. Kleinmann, J. Kołodyński, T. Kraft, J. Siewert, and G. Vitagliano for discussions. We acknowledge the support of the EU (ERC Starting Grant No. 258647/GEDENTQOPT, COST Action CA15220, QuantERA CEBBEC, QuantERA eDICT), the Spanish MCIU (Grant No. PCI2018-092896), the Spanish Ministry of Science, Innovation and Universities and the European Regional Development Fund FEDER through Grant No. PGC2018-101355-B-I00 (MCIU/AEI/ FEDER, EU), the Basque Government (Grant No. IT98616), and the National Research, Development and Innovation Office NKFIH (Grants No. K124351, No. KH129601, No. KH125096, and No. 2019-2.1.7ERA-NET-2020-00003). We also acknowledge support by the Foundation for Polish Science through IRAP project cofinanced by the EU within the Smart Growth Operational Programme (Contract No. 2018/MAB/5). G. T. thanks a Bessel Research Award of the Humboldt Foundation.

*toth@alumni.nd.edu http://www.gtoth.eu tvertesi@atomki.hu

[1] R. Horodecki, P. Horodecki, M. Horodecki, and K. Horodecki, Quantum entanglement, Rev. Mod. Phys. 81, 865 (2009); O. Gühne and G. Tóth, Entanglement detection, Phys. Rep. 474, 1 (2009); N. Friis, G. Vitagliano, M. Malik, and M. Huber, Entanglement certification from theory to experiment, Nat. Rev. Phys. 1, 72 (2019).

[2] L. Pezzé and A. Smerzi, Entanglement, Nonlinear Dynamics, and the Heisenberg Limit, Phys. Rev. Lett. 102, 100401 (2009).

[3] M. Gessner, L. Pezzè, and A. Smerzi, Resolution-enhanced entanglement detection, Phys. Rev. A 95, 032326 (2017). 
[4] P. Hyllus, W. Laskowski, R. Krischek, C. Schwemmer, W. Wieczorek, H. Weinfurter, L. Pezzé, and A. Smerzi, Fisher information and multiparticle entanglement, Phys. Rev. A 85, 022321 (2012); G. Tóth, Multipartite entanglement and high-precision metrology, Phys. Rev. A 85, 022322 (2012).

[5] B. Lücke, M. Scherer, J. Kruse, L. Pezzé, F. Deuretzbacher, P. Hyllus, J. Peise, W. Ertmer, J. Arlt, L. Santos, A. Smerzi, and C. Klempt, Twin matter waves for interferometry beyond the classical limit, Science 334, 773 (2011).

[6] R. Krischek, C. Schwemmer, W. Wieczorek, H. Weinfurter, P. Hyllus, L. Pezzé, and A. Smerzi, Useful Multiparticle Entanglement and Sub-Shot-Noise Sensitivity in Experimental Phase Estimation, Phys. Rev. Lett. 107, 080504 (2011).

[7] H. Strobel, W. Muessel, D. Linnemann, T. Zibold, D. B. Hume, L. Pezzé, A. Smerzi, and M. K. Oberthaler, Fisher information and entanglement of non-Gaussian spin states, Science 345, 424 (2014).

[8] P. Horodecki, Separability criterion and inseparable mixed states with positive partial transposition, Phys. Lett. A 232, 333 (1997).

[9] A. Peres, Separability Criterion for Density Matrices, Phys. Rev. Lett. 77, 1413 (1996).

[10] P. Horodecki, M. Horodecki, and R. Horodecki, Bound Entanglement can be Activated, Phys. Rev. Lett. 82, 1056 (1999).

[11] Ł. Czekaj, A. Przysiężna, M. Horodecki, and P. Horodecki, Quantum metrology: Heisenberg limit with bound entanglement, Phys. Rev. A 92, 062303 (2015).

[12] G. Tóth and T. Vértesi, Quantum States with a Positive Partial Transpose are Useful for Metrology, Phys. Rev. Lett. 120, 020506 (2018).

[13] P. Hyllus, O. Gühne, and A. Smerzi, Not all pure entangled states are useful for sub-shot-noise interferometry, Phys. Rev. A 82, 012337 (2010).

[14] In a general LOCC operation, large numbers of copies are used, and many rounds of classical communication take place. In our case no classical communication is needed, and in particular adding ancilla is a local operation (LO) which is an example of LOCC without classical communication (CC).

[15] M. Nawareg, S. Muhammad, P. Horodecki, and M. Bourennane, Superadditivity of two quantum information resources, Sci. Adv. 3, e1602485 (2017).

[16] M. Navascués and T. Vértesi, Activation of Nonlocal Quantum Resources, Phys. Rev. Lett. 106, 060403 (2011).

[17] C. Palazuelos, Superactivation of Quantum Nonlocality, Phys. Rev. Lett. 109, 190401 (2012).

[18] C. Helstrom, Quantum Detection and Estimation Theory (Academic Press, New York, 1976); A. Holevo, Probabilistic and Statistical Aspects of Quantum Theory (NorthHolland, Amsterdam, 1982); S. L. Braunstein and C. M. Caves, Statistical Distance and the Geometry of Quantum States, Phys. Rev. Lett. 72, 3439 (1994); D. Petz, Quantum Information Theory and Quantum Statistics (Springer, Berlin, Heilderberg, 2008); S. L. Braunstein, C. M. Caves, and G. J. Milburn, Generalized uncertainty relations: Theory, examples, and Lorentz invariance, Ann. Phys. (N.Y.) 247, 135 (1996).
[19] V. Giovannetti, S. Lloyd, and L. Maccone, Quantumenhanced measurements: Beating the standard quantum limit, Science 306, 1330 (2004); R. Demkowicz-Dobrzanski, M. Jarzyna, and J. Kolodynski, Chapter four-Quantum limits in optical interferometry, Prog. Opt. 60, 345 (2015); L. Pezze and A. Smerzi, Quantum theory of phase estimation, in Atom Interferometry (Proc. Int. School of Physics 'Enrico Fermi', Course 188, Varenna), edited by G. Tino and M. Kasevich (IOS Press, Amsterdam, 2014), pp. 691-741; G. Tóth and I. Apellaniz, Quantum metrology from a quantum information science perspective, J. Phys. A 47, 424006 (2014).

[20] L. Pezzè, A. Smerzi, M. K. Oberthaler, R. Schmied, and P. Treutlein, Quantum metrology with nonclassical states of atomic ensembles, Rev. Mod. Phys. 90, 035005 (2018).

[21] M. G. A. Paris, Quantum estimation for quantum technology, Int. J. Quantum. Inform. 07, 125 (2009).

[22] See the Supplemental Material at http://link.aps.org/ supplemental/10.1103/PhysRevLett.125.020402 for additional results on metrology with isotropic states and Werner states, as well as for metrology with bipartite pure entangled states. The Supplemental Material includes Refs. [23-35].

[23] K. Macieszczak, Zeno limit in frequency estimation with non-Markovian environments, Phys. Rev. A 92, 010102(R) (2015).

[24] I. Apellaniz, B. Lücke, J. Peise, C. Klempt, and G. Tóth, Detecting metrologically useful entanglement in the vicinity of Dicke states, New J. Phys. 17, 083027 (2015).

[25] F. Fröwis, M. Fadel, P. Treutlein, N. Gisin, and N. Brunner, Does large quantum Fisher information imply Bell correlations? Phys. Rev. A 99, 040101(R) (2019).

[26] G. Tóth and F. Fröwis (to be published).

[27] I. Apellaniz, M. Kleinmann, O. Gühne, and G. Tóth, Optimal witnessing of the quantum Fisher information with few measurements, Phys. Rev. A 95, 032330 (2017).

[28] H.-J. Sommers and K. Życzkowski, Statistical properties of random density matrices, J. Phys. A 37, 8457 (2004).

[29] G. Tóth, O. Gühne, and H. J. Briegel, Two-setting Bell inequalities for graph states, Phys. Rev. A 73, 022303 (2006).

[30] S. Popescu and D. Rohrlich, Generic quantum nonlocality, Phys. Lett. 166A, 293 (1992).

[31] M. Oszmaniec, R. Augusiak, C. Gogolin, J. Kołodyński, A. Acín, and M. Lewenstein, Random Bosonic States for Robust Quantum Metrology, Phys. Rev. X 6, 041044 (2016).

[32] D. M. Greenberger, M. A. Horne, and A. Zeilinger, Going beyond Bell's theorem, in Bell's Theorem, Quantum Theory, and Conceptions of the Universe, edited by M. Kafatos (Kluwer, Dordrecht, 1989), pp. 69-72.

[33] M. Krenn, M. Malik, R. Fickler, R. Lapkiewicz, and A. Zeilinger, Automated Search for New Quantum Experiments, Phys. Rev. Lett. 116, 090405 (2016).

[34] R. Uola, T. Kraft, J. Shang, X.-D. Yu, and O. Gühne, Quantifying Quantum Resources with Conic Programming, Phys. Rev. Lett. 122, 130404 (2019).

[35] T. Kraft, University of Siegen (private communication).

[36] M. Hotta and M. Ozawa, Quantum estimation by local observables, Phys. Rev. A 70, 022327 (2004).

[37] B. M. Escher, Quantum noise-to-sensibility ratio, arXiv: 1212.2533. 
[38] F. Fröwis, R. Schmied, and N. Gisin, Tighter quantum uncertainty relations following from a general probabilistic bound, Phys. Rev. A 92, 012102 (2015).

[39] K. Macieszczak, Quantum Fisher Information: Variational principle and simple iterative algorithm for its efficient computation, arXiv:1312.1356.

[40] K. Macieszczak, M. Fraas, and R. Demkowicz-Dobrzański, Bayesian quantum frequency estimation in presence of collective dephasing, New J. Phys. 16, 113002 (2014).

[41] M. A. Ciampini, N. Spagnolo, C. Vitelli, L. Pezzè, A. Smerzi, and F. Sciarrino, Quantum-enhanced multiparameter estimation in multiarm interferometers, Sci. Rep. 6, 28881 (2016).

[42] M. Horodecki and P. Horodecki, Reduction criterion of separability and limits for a class of distillation protocols, Phys. Rev. A 59, 4206 (1999).

[43] M. Horodecki, P. Horodecki, and R. Horodecki, General teleportation channel, singlet fraction, and quasidistillation, Phys. Rev. A 60, 1888 (1999).
[44] R. F. Werner, Quantum states with Einstein-Podolsky-Rosen correlations admitting a hidden-variable model, Phys. Rev. A 40, 4277 (1989).

[45] M. Huber, L. Lami, C. Lancien, and A. Müller-Hermes, High-Dimensional Entanglement in States with Positive Partial Transposition, Phys. Rev. Lett. 121, 200503 (2018).

[46] J. Bowles, M. T. Quintino, and N. Brunner, Certifying the Dimension of Classical and Quantum Systems in a Prepareand-Measure Scenario with Independent Devices, Phys. Rev. Lett. 112, 140407 (2014).

[47] N. Brunner, S. Pironio, A. Acin, N. Gisin, A. A. Méthot, and V. Scarani, Testing the Dimension of Hilbert Spaces, Phys. Rev. Lett. 100, 210503 (2008).

[48] R. Gallego, N. Brunner, C. Hadley, and A. Acín, Device-Independent Tests of Classical and Quantum Dimensions, Phys. Rev. Lett. 105, 230501 (2010).

[49] M. Navascués and T. Vértesi, Bounding the Set of Finite Dimensional Quantum Correlations, Phys. Rev. Lett. 115, 020501 (2015). 